BP-PP-3-7

\title{
Feasibility of using the homologous parietal peritoneum as a vascular substitute for venous reconstruction during abdominal surgery: An animal model
}

\author{
Seok-Hwan KIM*
}

Department of Surgery, Chungnam National University Hospital, Daejeon, Korea

Introduction: The interest in vascular substitutes has recently increased. We evaluated the feasibility of using a homologous parietal peritoneum (HPP) as a vascular substitute for venous reconstruction during abdominal surgery.

Methods: The inferior vena cava was replaced with an HPP after cross-linking with glutaraldehyde in 15 rabbits. At 7, 14, and 28 days, the patency rate, outer and inner graft diameters, histology, and immunohistochemistry were evaluated.

Results: Both the 7- and 14-day groups maintained vascular patency. Vascular patency was maintained in three rabbits in the 28-day group. The inner diameters of the anastomotic sites were $6.12 \pm 0.20 \mathrm{~mm}, 5.63 \pm 0.14 \mathrm{~mm}$, and $2.22 \pm 0.23 \mathrm{~mm}$ in the 7-day, 14-day, and 28-day groups, respectively. The mid-point inner diameters of the HPP grafts were $6.21 \pm 0.13 \mathrm{~mm}, 5.82 \pm 0.16 \mathrm{~mm}$, and $2.12 \pm$ $0.24 \mathrm{~mm}$ in each group, respectively. Endothelial cell proliferation on the HPP graft surfaces in all groups was based on the histological findings from the first group. Multiple neo-vascularization of the HPP graft were found in the 14- and 28-day groups, indicating neo-media formation. Acute inflammation appeared to progress to the entire layer of the HPP graft without an intraluminal thrombus, but the graft was patent in the 14-day group. In the 28-day group, two rabbits showed near-total occlusion and a thrombus formed in the HPP graft at the anastomosis site with severe stricture; however, the rabbits were alive and had collateral vessel formation.

Conclusions: Use of the HPP is feasible for venous reconstruction in abdominal surgery. 